\title{
Skin prick test analysis reveals cross-sensitization to tomato profilin and grass pollen in nasobronchial- allergic patients with history of tomato food allergy
}

\author{
${ }^{1}$ Department of Biochemistry and Nutrition, CSIR - Central Food Technological Research Institute (CFTRI), Mysuru, \\ Karnataka, India \\ ${ }^{2}$ Department of Radiation Oncology, The Houston Methodist Research Institute, Houston, TX 77030, U.S.A. \\ ${ }^{3}$ Allergy Asthma Associates, K. M. Puram, Mysuru, Karnataka, India
}

\section{KEY WORDS}

cross-sensitization; grass pollen;

profilin; respiratory allergy; tomato

\author{
Corresponding author \\ Yeldur P. Venkatesh \\ Department of Biochemistry and Nutrition \\ CSIR-CFTRI, KRS Road, Mysuru-570020 \\ Karnataka State, India \\ Phone: +91 8212546991 \\ Fax: +91 8212517233 \\ E-mail: venkatyp@yahoo.com
}

Doi

10.23822/EurAnnACI.1764-1489.27

\begin{abstract}
Summary
The association between grass pollen sensitization and food allergy to tomato is of great interest. We report here, the first such study in Indian population.

We investigated 246 allergic rhinitis / asthma patients by diagnostic case history and skin prick test (SPT); grass pollen mix, tomato extract and purified tomato profilin were used for SPT. Tomato profilin was purified by affinity chromatography, and analyzed by HPLC (95\% purity) and SDS-PAGE (14 kDa).

We observed that $38 \%$ of the patients had sensitization to both grass pollen and tomato fruit, of which 92\% were sensitized to tomato profilin. Among patients with a history of food allergy to tomato fruit, the association was more pronounced (66\%).

Tomato profilin appears to be an important cross-sensitizing panallergen in respiratory allergic patients in the Indian subcontinent.
\end{abstract}

\section{Abbreviations}

OAS, oral allergy syndrome; PBS, phosphate-buffered saline; PLP, poly-L-proline; SPT, skin prick test.

\section{Introduction}

Allergic rhinitis is an inflammation of the mucous membranes that occurs when allergens to which the patient is previously sensitized, come in contact with the lining of the nose. It is characterized by sneezing, congestion, itching and runny nose, and itchy, watery eyes. The common allergens causing allergic rhinitis are house dust, pollens from weeds, grasses and trees, fungi and mold spores, animal dander and some foods. Seasonal allergic rhinitis (SAR; hay fever) affects 70 million people around the globe $(1,2)$. It is the most common allergic disease encountered in the clinics and constitutes $-55 \%$ of all allergies seen in India (3). People who suffer from perennial rhinitis have symptoms year round, usually triggered by dust mites and cockroach droppings, indoor molds, and/or animal dander (1-3). Asthma is a chronic inflammatory disease of the airways associated with recurrent episodes of bronchoconstriction and increased bronchial hyper-responsiveness $(3,4)$. In susceptible individuals, this inflammation causes recurrent bouts of wheezing, chest tightness, and cough; those with asthma often have atopy as a significant predisposing factor. Nearly $80 \%$ of all asthmatics also have allergies and their symptoms are triggered by specific allergens. Asthma is known to commonly co-occur with allergic rhinitis (1-4).

The association between respiratory allergies and food allergy has been established (5-8). Complex food allergy syndromes 
involving allergy to fresh fruits / vegetables and sensitization to inhalant pollen allergens are commonly encountered in allergy clinics, sometimes extending over a range of botanically unrelated plant species (9-11). Typically, these patients experience itchy mouth, tongue (oropharyngeal pruritus), and glottic edema, commonly recognized as pollen-food allergy syndrome or oral allergy syndrome (OAS) (12); it has been shown that $-2-4 \%$ of European adults experience this syndrome. OAS is caused by sensitization to cross-reactive determinants shared by various pollens and different fruits / vegetables. Well-known examples of such cross-allergenicity are: (i) birch pollen (apple, hazelnut, carrot, potato, celery, kiwi fruit, etc), (ii) mugwort pollen (celery, carrot, spices), (iii) grass pollen (melon, tomato, peanut, watermelon, orange), and (iv) ragweed pollen (melon, watermelon, banana, zucchini, cucumber). Approximately 35\% of patients with such types of pollinosis show hypersensitivity to antigenically-related fruits and vegetables (9-12).

Profilins $(12-15 \mathrm{kDa})$ are a family of highly conserved plant pan-allergens (13-15). The ubiquitous occurrence of profilins in distantly-related plants provides a molecular basis for the frequently observed phenomenon of cross-sensitization towards unrelated plant species in allergic patients. Profilin was first identified as an allergen in birch pollen, and has since been shown to be present as a multigene panallergen family in many plants (13-15); it is described as a pan-allergen, because it was shown to be recognized by the serum IgE of $>20 \%$ of pollen-allergic patients (13-15). Profilins from tree, grass and weed pollens, many fruits and vegetables, and latex have been identified as cross-reactive allergens, and studied extensively (16-18).

Profilin binds to poly-(L-proline) (PLP) with high affinity (1921 ), a property that is exploited in the purification of many profilins (16-18). The amino acid sequence of profilins is not very highly conserved across many phyla (protozoa, mammalian, yeast, insect, echinoderm and plant); despite this, the structure is conserved $(22,23)$. Possibly connected with the sequence diversity, ubiquity, and abundance of profilins in eukaryotes is the fact that plant profilins are important cross-reactive allergens, and are identified as pan-allergens (23).

The prevalence of tomato (Solanum lycopersicum L.) allergy was estimated to range from $1.5 \%$ to $16 \%$ among food-allergic populations (24-27), and up to $39.2 \%$ among grass pollen-allergic patients (28). Profilin from tomato fruit (14 kDa; Sola 11 ) has been identified as a food allergen, and has been cloned and characterized (29,30). It was observed at the Allergy, Asthma and Chest Centre in Mysuru (India) that most of the allergic rhinitis and/or asthma patients (patients sensitized to pollen) had allergy to one or more raw vegetables and fruits. The major symptoms were triggering or worsening of allergic rhinitis / asthma, and OAS. Tomato, banana, cucumber, and citrus fruits were the most common offending foods. The association between grass pollen sensitization and food allergy to tomato fruit is known, and the heat-labile cross-reactive allergen profilin has been implicated in such cases. Until now, studies have not been reported from India with respect to tomato allergy in pollen-allergic patients, and the importance of profilin. The present study was undertaken to investigate the spectrum of cross-allergenicity between tomato and grass pollen among allergic rhinitis / asthma patients, and to assess whether tomato profilin is a cross-reactive allergen in a representative population from Mysuru city in South India.

\section{Materials and methods}

\section{Reagents and allergenic extracts}

Poly-(L-proline) (average mol. wt. 5000 Da; PLP), urea, DMSO (dimethyl sulfoxide), phenylmethanesulfonyl fluoride (PMSF) and trifluoroacetic acid (TFA) were products of Sigma-Aldrich Chemical Co., St Louis, MO, USA. Sepharose-4B was obtained from Pharmacia, Uppsala, Sweden. Cyanogen bromide (CNBr) was a product of Sisco Research Laboratories, Mumbai, India. Grass pollen mix extracts were obtained from Bayer Corp., Spokane, WA, USA and Greer Laboratories, Lenoir, NC, USA. Southern grass pollen mix (\#1651, Bayer Corp.) contained pollens from Bermuda, Johnson, Kentucky blue, Orchard, Redtop, sweet Vernal, and timothy grasses. Grass pollen mix (\#P28, Greer Laboratories) contained pollens from Bermuda, Johnson, Kentucky blue, Orchard, Redtop, Timothy, sweet Vernal meadow, fescue, and perennial rye grasses. All other chemicals and reagents were of analytical grade.

\section{Preparation of tomato extract}

A $50 \% \mathrm{w} / \mathrm{v}$ tomato extract was prepared by blending ripe tomato fruits in a blender using cold phosphate-buffered saline (PBS), and filtering the extract to obtain a clear filtrate. The filtrate thus obtained was dialyzed against PBS using $3500 \mathrm{MWCO}$ dialysis membrane, sterile filtered, and used for SPT. For the purification of profilin, tomato extract was prepared in $10 \mathrm{mM}$ Tris- $\mathrm{HCl}$ buffer, $\mathrm{pH} 7.8$ containing $0.1 \mathrm{M} \mathrm{KCl}, 0.1 \mathrm{M}$ glycine, $0.2 \mathrm{mM}$ PMSF, $0.5 \mathrm{mM}$ dithiothreitol, and $0.2 \%$ sodium azide (buffer $A$ ). Protein content was determined by the method of Bradford (31).

\section{Allergic subjects and case history}

Subjects diagnosed as having allergic rhinitis or bronchial asthma or both, based on diagnostic tests amongst the incoming subjects at the Allergy, Asthma and Chest Centre, Mysuru and at the 'Asthma and Allergy' medical camps conducted by the center in and around Mysore city (over a period of 2 calendar years) were enrolled in this study. 
A detailed case history was obtained for each subject based on a questionnaire which mainly included age, family history of allergic diseases, history and symptoms of respiratory allergy, history of food allergy, symptoms of allergy to tomato and/or any other foods, any other allergic symptoms, onset, frequency and status of symptoms, diagnostic tests undergone earlier and medications taken, if any.

All procedures performed and studies involving human participants were approved by the institutional research ethics committee, and were conducted in accordance with the ethical standards established in the Declaration of Helsinki of 1946 and its later amendments or comparable ethical standards. Informed consent was obtained from all participants before enrollment in the study.

\section{Preparation of poly-(L-proline)-Sepharose-4B affinity matrix}

The preparation of activated Sepharose $4 \mathrm{~B}$ was performed by the cyanogen bromide activation method described in the literature (32). Briefly, $30 \mathrm{~mL}$ of slurry of washed agarose beads (Sepharose $4 \mathrm{~B}$ ), consisting of equal volumes of gel and water, was added to $30 \mathrm{~mL}$ of $2 \mathrm{M}$ sodium carbonate and mixed by stirring slowly. The rate of stirring was increased and $1.5 \mathrm{~mL}$ of an acetonitrile solution of cyanogen bromide ( $2 \mathrm{~g}$ cyanogen bromide per $\mathrm{mL}$ of acetonitrile) was added all at once. The slurry was stirred vigorously for 1-2 min, after which the slurry was poured on to a sintered glass funnel, washed sequentially with $300 \mathrm{~mL}$ each of $0.1 \mathrm{M}$ sodium bicarbonate $(\mathrm{pH} 9.5)$, water, and coupling buffer $\left(0.1 \mathrm{M} \mathrm{KHCO}_{3}, \mathrm{pH} 8.3\right.$, containing $0.5 \mathrm{M}$ $\mathrm{KCl})$. The coupling of PLP was done according to the method of Lindberg et al (19). After the last wash, the slurry was filtered under vacuum to a moist, compact cake and transferred to a plastic bottle containing the ligand solution; the ligand solution contained $100 \mathrm{mg}$ of PLP (average mol. wt. 5000) dissolved in $30 \mathrm{~mL}$ coupling buffer. The coupling mixture was incubated at room temperature for $2 \mathrm{~h}$ and continued at $4{ }^{\circ} \mathrm{C}$ overnight with gentle stirring. The coupling was followed by recording the absorbance at $230 \mathrm{~nm}$; after completion of the reaction, about $50-60 \%$ of PLP had bound to the matrix. Uncoupled ligand was removed by washing the gel in coupling buffer, after which the remaining active groups on Sepharose were deactivated by incubating with $0.1 \mathrm{M}$ Tris- $\mathrm{HCl}$ buffer, $\mathrm{pH}$ 8.0.

\section{Purification of tomato profilin by affinity chromatography}

The purification of tomato profilin by affinity chromatography was carried out as described in the literature $(20,21)$. The poly-(L-proline)-Sepharose $4 \mathrm{~B}$ affinity matrix was packed in to a glass column $(1.6 \mathrm{~cm}$ i.d. $\times 7.5 \mathrm{~cm})$ and was equilibrated with buffer $A$ (described under "Preparation of tomato extract"). Tomato extract was passed through the column at $4{ }^{\circ} \mathrm{C}$, at a flow rate of $25 \mathrm{~mL} / \mathrm{h}$. The column was then washed with buffer $A$ until the absorbance at $280 \mathrm{~nm}$ of the effluent decreased to that of the buffer. The bound profilin was eluted using buffer $A$ containing either 30\% DMSO, or $7 \mathrm{M}$ urea (after washing initially with $3 \mathrm{M}$ urea). Profilin-containing fractions were pooled, dialyzed, concentrated by lyophilization, and stored in aliquots at $-20^{\circ} \mathrm{C}$. The purity of the purified tomato profilin was determined by SDS-PAGE and reverse-phase HPLC.

\section{Skin prick tests}

Glycerinated (50\%) allergen extracts were used for SPT by following the standard procedure (33) using sterile prick lancetter (Bayer Pharmaceutical Division, Spokane, WA, USA). Affinity-purified tomato profilin was used at $100 \mu \mathrm{g} / \mathrm{mL}$ concentration in glycerinated PBS. Glycerinated PBS ( $\mathrm{pH} 7.4$ ) and histamine phosphate $(1.4 \mathrm{mg} / \mathrm{mL}$, equivalent to $1 \mathrm{mg} / \mathrm{mL}$ histamine base) served as negative and positive controls, respectively. The diameter of the wheal was read after $20 \mathrm{~min}$. SPT was graded based on the diameter of wheal produced by a sample in comparison to that of positive control $(\mathrm{H}): 1+$ (less than $1 / 2 \mathrm{H}) ; 2+$ (equal to or greater than $1 / 2 \mathrm{H}$ ); $3+$ (equal to or greater than $\mathrm{H}$ ); $4+$ (equal to or greater than $2 \mathrm{H}$ ). The test was considered negative if the wheal diameter was equal to or less than that of the negative control $(3 \mathrm{~mm})$.

\section{Results}

\section{Demographic analysis}

A panel of 246 subjects (age range: 4-80 years) who had allergic rhinitis or asthma or both, were investigated by careful case history and SPT in order to understand the prevalence of grass pollen sensitization and tomato allergy, and the relevance of tomato profilin as a cross-reactive allergen among such a population. The number of subjects in different age groups based on gender is shown in Figure 1 (panel a); nearly 70\% had family history of allergy (Figure 1, panel b). The prevalence of clinical symptoms among the 246 subjects is shown in Figure 2 (panel a). Wheeze was most prevalent; nasal symptoms (itchy nose and rhinorrhea) and pharyngeal symptoms (cough, tightness and itching in the oropharyngeal region) were also common. Among the cutaneous symptoms, urticaria was the most common. Considering the actual respiratory disease, $66 \%$ of subjects in the study group had allergic rhinitis and $60 \%$ of them had asthma, while $43 \%$ of subjects suffered from both allergic rhinitis and asthma (Figure 2, panel b).

The demographic and clinical symptoms of allergic rhinitis / asthma patients having a history of tomato allergy $(n=74)$ are presented in Table I. Majority of the subjects, who had histo- 
Figure 1- Panel $\boldsymbol{a}$, distribution of subjects of the study group into different age groups and sex ( $n=246) ;$ panel $\boldsymbol{b}$, family history of allergy in the study group based on case history $(n=246 ;+v e$, positive; -ve, negative).
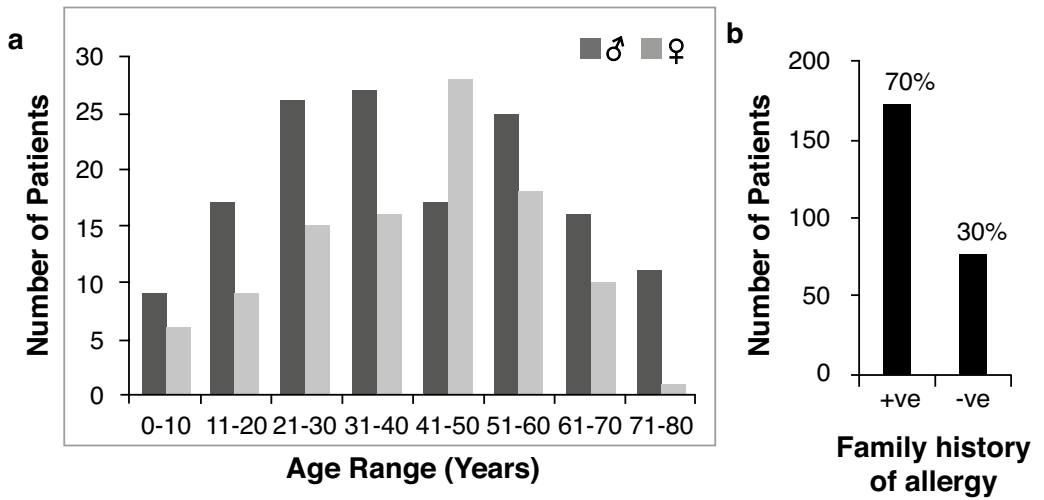

ry of adverse reactions to tomato, had experienced triggering or worsening of respiratory symptoms after eating raw tomato fruit $(56 \%)$. The majority of the patients $(-66 \%)$ had nasal / pharyngeal symptoms; patients with wheezing were even higher (74\%). Other symptoms of allergy to tomato were OAS (28\%), and rarely, skin rashes or urticaria. Other major offending foods in this study population are banana (57\%), citrus fruits (40\%), cucumber (32\%) and eggplant (8\%).

Figure 2 - Panel a, symptoms of respiratory and cutaneous allergy in the study group $(n=246)$ based on case history; panel $\boldsymbol{b}$, prevalence of allergic rhinitis and asthma in the study group $(n=246)$ based on case history.

a

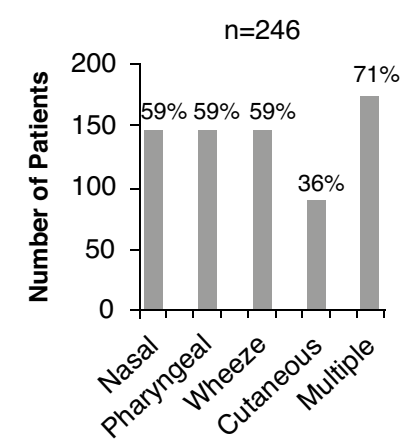

b

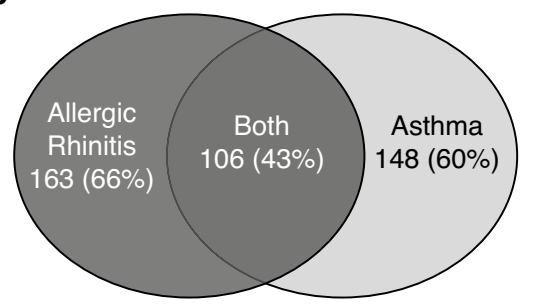

Table I - Demographic characteristics of subjects with allergic rhinitis / asthma reporting history of allergy to tomato fruit.

\begin{tabular}{ll}
\hline Feature & Number or percentage \\
\hline patients (n) & 74 \\
\hline age (y) & $5-80$ \\
\hline median age (y) & 40.5 \\
\hline male & $27(36 \%)$ \\
\hline female & $46(64 \%)$ \\
\hline family history of allergy & $26(35 \%)$ \\
\hline Symptoms & $49(66 \%)$ \\
\hline nasal & $48(65 \%)$ \\
\hline pharyngeal & $55(74 \%)$ \\
\hline wheeze & $22(30 \%)$ \\
\hline cutaneous (urticaria) & $10(13 \%)$ \\
\hline multiple & \\
\hline Other offending foods & $42(57 \%)$ \\
\hline Major & $30(40 \%)$ \\
\hline banana & $24(32 \%)$ \\
\hline citrus fruits & $6(8 \%)$ \\
\hline cucumber & $<1 \%$ \\
\hline eggplant & \\
\hline Minor & \\
\hline $\begin{array}{l}\text { apple, coconut, guava, grapes, } \\
\text { mango, milk, onion, pulses, } \\
\text { wheat, yam }\end{array}$ & \\
\hline
\end{tabular}


Affinity purification of tomato profilin from tomato extract for SPT studies

The elution profiles of affinity chromatography of tomato fruit extract on poly-(L-proline)-Sepharose- $4 \mathrm{~B}$ carried out to purify tomato profilin are presented in Figure 3. Two different eluants, viz., 30\% DMSO (Figure 3, panel a) and $7 \mathrm{M}$ urea (Figure 3, panel b) were used in separate experiments to elute the bound tomato profilin. The fractions were analyzed by SDS-PAGE, and the fractions containing the $14 \mathrm{kDa}$ protein were pooled, dialyzed using $3500 \mathrm{MWCO}$ dialysis membrane, and concentrated. The SDS-PAGE patterns of the affinity chromatographic eluates are shown in Figure 4. Elution using $7 \mathrm{M}$ urea yielded highly pure $14 \mathrm{kDa}$ protein (Figure 3, panel b), whereas the DMSO eluate showed the presence of other contaminating proteins around 10,20 , and $66 \mathrm{kDa}$ regions in SDS-PAGE, besides the protein band at $-14 \mathrm{kDa}$ (Figure 3, panel a). It is known that DMSO elutes both profilin and profilactin complexes, whereas $7 \mathrm{M}$ urea (after the initial washing with $3 \mathrm{M}$ urea) elutes profilin selectively (20). The

Figure 3 - Affinity chromatography of 50\% w/v tomato extract (500 mL) on poly-(L-proline)-Sepharose-4B column $(1.6 \mathrm{~cm}$ i.d. $\times$ $7.5 \mathrm{~cm}$ ) for the preparation of tomato profilin. Temperature, $4^{\circ} \mathrm{C}$; flow rate, $25 \mathrm{~mL} / \mathrm{h}$; fraction size, $2 \mathrm{~mL} /$ fraction. Panel a, elution using 30\% DMSO; fractions 16-19 containing the $14 \mathrm{kDa}$ protein were pooled. Panel $\boldsymbol{b}$, elution using $7 M$ urea after washing with 3 $M$ urea; fractions 4-8 containing the $14 k D a$ protein were pooled.

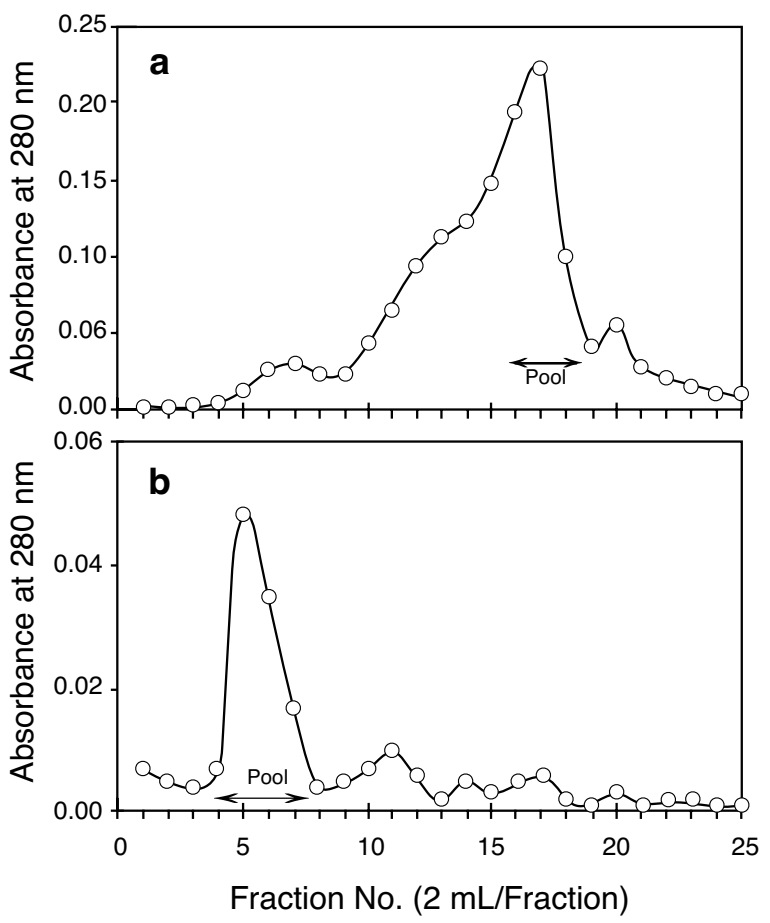

Figure 4 - SDS-PAGE analyses of affinity-purified tomato profilin. Panel a, $15 \%$ gel (reducing). $M$, mol. wt. markers; lane 1, tomato profilin eluted using 30\% DMSO. Panel b; $12 \%$ gel (reducing). Lane 1, tomato whole extract; $M$, mol. wt. markers; lane 2, tomato profilin eluted using $7 M$ urea. Panel $\boldsymbol{c}$, reverse-phase HPLC profile of affinity-purified tomato profilin (eluted using $7 \mathrm{M}$ urea). Column: Zorbax C8, DuPont, Bellefonte, PA, U.S.A.; elution: binary gradient of $0.1 \%$ TFA (solvent $A$ ), and $70 \%$ acetonitrile $+0.05 \%$ TFA (solvent B); flow rate, $1 \mathrm{~mL} / \mathrm{min}$; UV detection, $280 \mathrm{~nm}$. The pure protein showed an $R T$ (retention time) of $39 \mathrm{~min}$.

a
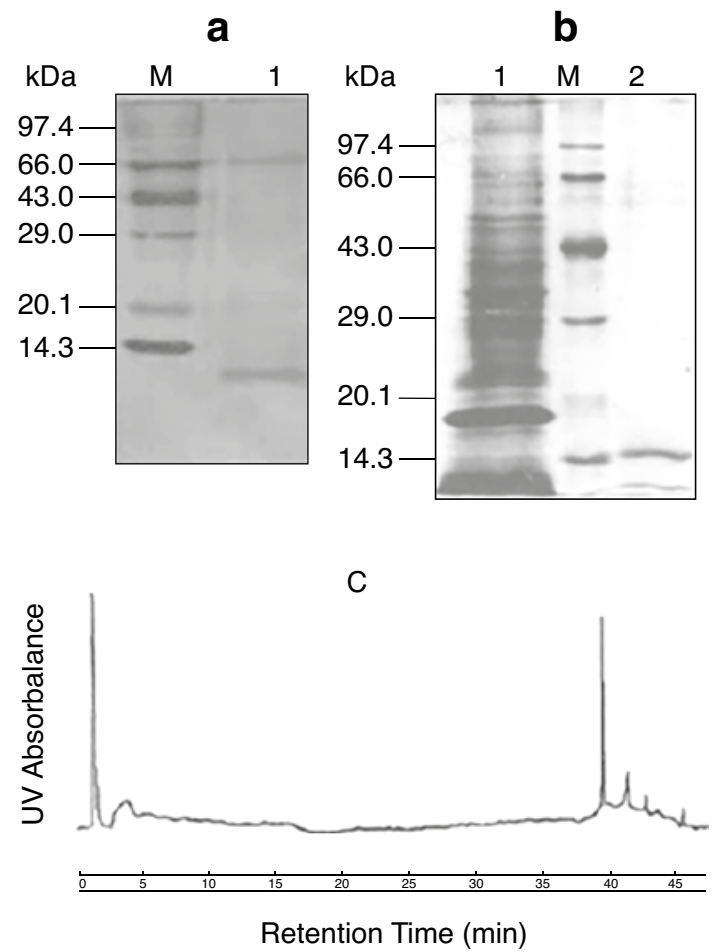

homogeneity of urea-eluted tomato profilin was determined by reverse-phase HPLC analysis where it showed $-95 \%$ purity (Figure 4, panel c).

Studies on the relevance of tomato profilin as a cross-reactive allergen in patients with respiratory allergy

In the study group of 246 allergic rhinitis / asthma subjects, sensitization to grass pollens, tomato fruit and affinity-purified tomato profilin was determined by SPT. As high as $182(74 \%)$ subjects showed sensitization to grass pollen extract (Figure 5, panel a); sensitization to tomato fruit extract and tomato profilin was $43 \%$ and $51 \%$, respectively. Out of 246 subjects, 95 (38\%) showed positive SPT with both grass pollen mix and tomato extracts (Figure 5, panel b). If one considers only 
the grass-pollen sensitized subjects, the percentage of subjects reactive to tomato extract comes to $-54 \%$. Out of the 95 subjects who showed cross-sensitization to both grass pollen and tomato extracts, 82 subjects ( $92 \%$ of subgroup) showed positive SPT with affinity-purified tomato profilin (Figure 5, panel b). These results based on SPT indicate that cross-sensitization to grass pollen and tomato fruit is very prevalent at $38 \%$ among allergic rhinitis / asthma patients, and majority of them $(92 \%)$ are sensitized to the cross-reactive panallergen, tomato profilin.

SPT studies in the subgroup of allergic rhinitis / asthma patients with a history of adverse reactions to tomato fruit

In this subgroup of 74 subjects, SPT with grass pollen mix extract, tomato fruit extract, and affinity-purified tomato profilin was positive in $84 \%, 67 \%$, and $64 \%$ of subjects, respectively (Figure 6); the percentage of patients giving a $2+$ reaction in SPT with all the 3 extracts was higher compared to those showing $1+$ or $3+$ reaction.

Out of $48(65 \%)$ subjects who showed cross-sensitization to tomato fruit and grass pollen, 44 (91\%) subjects showed sensitization to tomato profilin (Figure 7). These data indicate that tomato profilin is a very important cross-sensitizing allergen among the tomato allergens in allergic rhinitis / asthma patients sensitized to grass pollen and with a history of tomato allergy.

Figure 5 - Pattern of sensitization to grass pollen, tomato fruit, and tomato profilin based on SPT. Panel $\boldsymbol{a}$, the entire study group of allergic rhinitis / asthma patients $(n=246)$. Panel $\boldsymbol{b}$, subgroup of patients cross-sensitized to grass pollen and tomato fruit as determined by positive SPT.

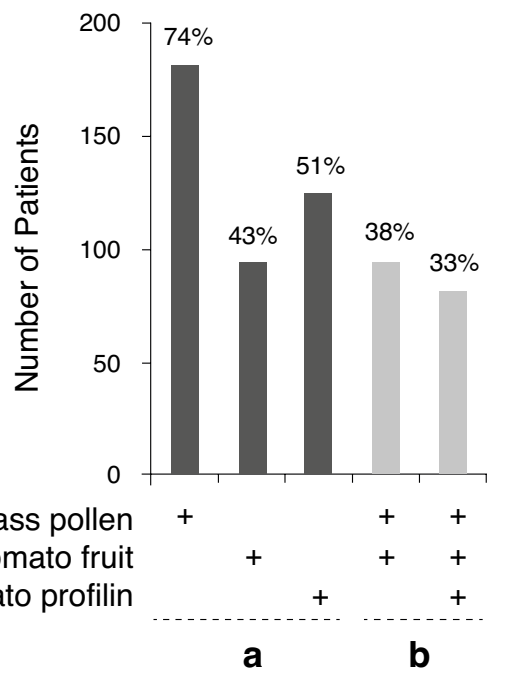

Figure 6 - Gradation of SPT in the subgroup of allergic rhinitis I asthma patients with history of adverse reactions to tomato fruit ingestion $(n=74)$. SPT was graded based on the diameter of wheal produced by a sample in comparison with that of positive control (histamine, $H$ ): $1+$, less than $1 / 2 H ; 2+$, equal to or greater than $1 / 2 \mathrm{H} ; 3+$, equal to or greater than $H ; 4+$, equal to or greater than 2H. SPT was considered negative if the wheal diameter was equal to or less than that of the negative control.

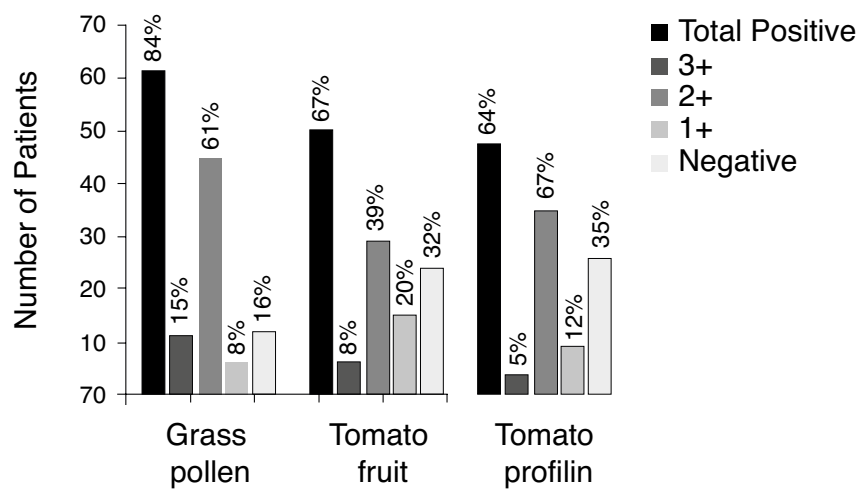

Figure 7 - Pattern of sensitization to grass pollen, tomato fruit and tomato profilin based on SPT. Panel a, allergic rhinitis / asthma subjects with history of adverse reactions to tomato fruit ingestion $(n=74)$; and panel $\boldsymbol{b}$, those with positive SPT to grass pollen and tomato fruit.

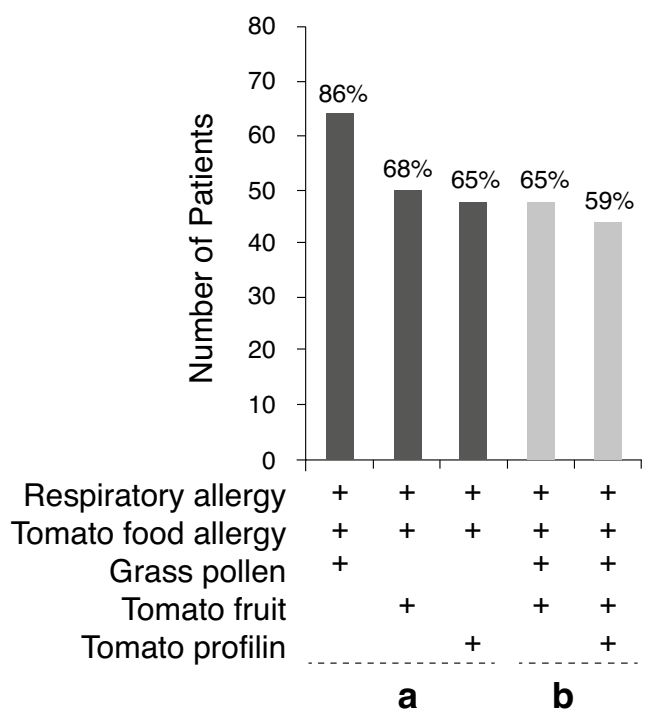

\section{Discussion}

In this study, we have evaluated the role of tomato profilin in presenting cross-reactivity with grass pollen-sensitized allergic subjects having respiratory allergy. Petersen et al (25) identified the main tomato allergens using sera from 8 subjects with high 
specific $\operatorname{IgE}$ to tomatoes that also had grass pollen allergies; 5 out of 8 sera reacted to $\beta$-fructofuranosidase $(\beta-F F)$, and another 5 reacted to profilin. Foetish et al (28) studied the relevance of tomato allergy in pollen-allergic patients in a group of 32 birch pollen-allergic patients with history of adverse reactions to tomato fruit, and found that tomato allergy occurred with a prevalence of $9 \%$ in the study group, and the majority of pollen-associated allergies to tomato were due to ubiquitous allergenic structures such as profilin (44\%) and cross-reactive IgE binding $N$-glycans (35.5\%). However, in the present study the sensitization was estimated based only on SPT, in which a positive result shows the presence of mast cell-bound specific $\mathrm{IgE}$ to a particular extract or protein. Despite SPT producing false positive and false negative tests with about $5-10 \%$ margin of error, it is a simple and reasonably accurate test most widely used in allergy clinics as the first diagnostic test (33). A number of impactful studies have demonstrated the sensitivity of prick tests as better or comparable to in vitro tests or immunoassays for the detection of specific IgE using whole extracts or purified allergens, and have positively confirmed their reliability to assess allergen sensitization (34-38). Although not supported by data on serum allergen-specific IgE, the results presented here give an idea of the pattern of sensitization to tomato profilin in pollen-allergic patients in a South Indian city.

Tomato fruit profilin gene has been cloned and the recombinant protein was shown to be IgE-reactive with a prevalence of $22 \%$ in tomato-allergic patients (29). In subjects with tomato allergy and multiple sensitization to other foods and birch pollen, $\mathrm{IgE}$ directed against tomato profilin showed a strong cross-reactivity with profilins from plant food sources and birch pollen. Westphal et al (30) concluded that tomato profilin is a minor allergen in tomato fruit. In studies of profilin sensitization by SPT, Asero et al $(34,35)$ highlighted the clinical importance of diagnosing hypersensitivity to single food allergenic proteins immediately in the office; this is especially useful for the allergist if the relevant food allergen sources contain several allergenic proteins that show different physicochemical characteristics and, hence, completely different risk profiles. The large majority of profilin-allergic patients reported OAS as the only food-induced symptom, and was able to tolerate the offending foods if they were cooked or processed. Profilin should be considered a clinically relevant food allergen, and allergy to melon, watermelon, tomato, banana, pineapple and orange may be considered as a marker of profilin hypersensitivity $(34,35)$.

In evaluating the clinical features and usefulness of current diagnostic methods for tomato allergy, Asero (39) found that tomato allergy was detected in $33 \%$ of subjects with plant food allergy, and was significantly associated with profilin hypersensitivity $(p<0.001)$, and concluded that tomato allergy occurs by sensitization towards different proteins; most cases are seen in profilin-hypersensitive subjects and are mild. In another study of 26 patients overexposed to grass who were referred for severe food reactions, oral provocation with purified profilin resulted in a significant number of patients showing severe positive food challenge test reactions at low doses of profilin (40). These investigators claim that profilins are complete food allergens in food-allergic patient populations that are exposed to high levels of grass pollen; this patient type constitutes an optimal model to understand the link between respiratory and food allergies. It is likely that intake through the oral mucosa might be a relevant route of exposure to food allergens.

\section{Conclusions}

The association between sensitization to pollen and allergy to raw vegetables and fruits is well established (5,9-11,13-15,18). Understanding the spectrum of cross-reactive pollen-food allergies in different populations is required for the proper diagnosis and treatment of such allergic disorders. The association between grass-pollen sensitization and allergy to tomato fruit is known from studies in Europe and Japan (24-26,28,30); however, there are no reports of such studies in the Indian population. The present study describes a preliminary investigation based on case history and SPT in a study group of 246 allergic rhinitis / asthma patients in Mysuru city, India, aimed at understanding the importance of tomato profilin as a cross-reactive allergen. Tomato profilin was purified by affinity chromatography, and was used in SPT along with tomato fruit extract and grass pollen mix extracts. The results showed that $38 \%$ of subjects had sensitization to both tomato fruit and grass pollen, out of which $92 \%$ were sensitized to tomato profilin. In the subgroup of allergic rhinitis / asthma patients with a history of adverse reaction to raw tomato fruit $(32 \%)$, the result was even more pronounced, with $66 \%$ subjects showing cross-sensitization to tomato fruit and grass pollen, out of which majority of them (91\%) showed positive SPT with tomato profilin. Our results clearly indicate that tomato profilin is a very important cross-sensitizing allergen in allergic rhinitis / asthma patients sensitized to grass pollen, and more so in patients with history of food allergy to tomato fruit. In conclusion, it may be advantageous to incorporate purified profilins (e.g., tomato profilin) in the routine SPT procedures for patients with respiratory allergy, and subsequently, in specific immunotherapy protocols in allergy clinics for desensitization of allergic subjects sensitized to profilin.

\section{Conflict of interest}

The authors declare that they have no conflict of interest.

\section{Acknowledgments}

We acknowledge the Council of Scientific and Industrial Research (CSIR), New Delhi, for the funding of the institutional 
project (MLP-1407) to YPV and senior research fellowship to VLH. The sponsor (CSIR, New Delhi) did not have any involvement in the study design, collection, analysis and interpretation of data, in writing the report and in the decision to submit the article for publication.

\section{References}

1. Busse W, Holgate S (editors). Asthma and rhinitis. Boston: Blackwell Scientific Publications; 1995.

2. Braido F, Arcadipane F, Marugo F, Hayashi M, Pawankar R. Allergic rhinitis: current options and future perspectives. Curr Opin Allergy Clin Immunol 2014; 14(2):168-76.

3. Shaikh WA (editor). Allergy and asthma: a tropical view. New Delhi: IJCP Group; 2000.

4. Shah R, Saltoun CA. Chapter 14: Acute severe asthma (status asthmaticus). Allergy Asthma Proc 2012; 33(Suppl 1):S47-S50.

5. Rumsaeng V, Metcalfe DD. Asthma and food allergy. Nutr Rev. 1998; 56(1 Pt 2):S153-60.

6. Brook G, Loots J. The role of foods in asthma. In: The internet journal of nutrition and wellness, 2005; 2(2):1-10. http://www. ispub.com/IJNW/2/2/4665.

7. Allan K, Devereux G. Diet and asthma: nutrition implications from prevention to treatment. J Am Diet Assoc 2011; 111(2):258-68.

8. Varraso R. Nutrition and asthma. Curr Allergy Asthma Rep 2012; 12(3):201-10.

9. Eriksson NE, Formgren H, Svenonius E. Food hypersensitivity in patients with pollen allergy. Allergy 1982; 37(6):437-43.

10. Calkhoven PG, Aalberse M, Koshte VL, Pos O, Oei HD, Aalberse RC. Cross-reactivity among birch pollen, vegetables and fruits as detected by $\operatorname{IgE}$ antibodies is due to at least three distinct cross-reactive structures. Allergy 1987; 42(5):382-90.

11. Sicherer SH. Clinical implications of cross-reactive food allergens. J Allergy Clin Immunol 2001; 108(6):881-90.

12. Webber CM, England RW. Oral allergy syndrome: a clinical, diagnostic, and therapeutic challenge. Ann Allergy Asthma Immunol 2010; 104(2):101-8; quiz 109-10, 117.

13. Valenta R, Duchene M, Pettenbeurger K, Sillaber C, Valent P, Bettelheim $\mathrm{P}$, et al. Identification of profilin as a novel pollen allergen: IgE cross-reactivity in sensitized individuals. Science 1991; 253(5019):557-60.

14. van Ree R, Voitenko V, van Leeuwen WA, Aalberse RC. Profilin is a cross-reactive allergen in pollen and vegetable foods. Int Arch Allergy Immunol 1992; 98(2):97-104.

15. Valenta R, Duchene M, Ebner C, Valent P, Sillaber C, Deviller P, et al. Profilins constitute a novel family of functional plant pan-allergens. J Exp Med 1992; 175(2):377-85.

16. Vrtala S, Wiedemann P, Mittermann I, Eichler HG, Sperr WR, Valent P, et al. High-level expression in Escherichia coli and purification of recombinant plant profilins: comparison of IgE-binding capacity and allergenic activity. Biochem Biophys Res Commun 1996; 226(1):42-50.

17. Asturias JA, Arilla MC, Gomez-Bayon N, Martinez J, Martinez A, Palacios R. Cloning and high level expression of Cynodon dactylon (Bermuda grass) pollen profilin (Cyn d 12) in Escherichia coli. Purification and characterization of the allergen. Clin Exp Allergy 1997; 27(11):1307-13.

18. Scheurer S, Wangorsch A, Nerkamp J, Skov PS, Ballmer-Weber B, Wuthrich B, et al. Cross-reactivity within the profilin panallergen family investigated by comparison of recombinant profilins from pear (Pyr c 4), cherry (Pru av 4) and celery (Api g 4) with birch pollen profilin Bet v 2. J Chromatogr B Biomed Sci Appl 2001; 756(1-2):315-25.

19. Lindberg U, Schutt CE, Hellsten E, Tjader A-C, Hult T. The use of poly(L-proline)-Sepharose in the isolation of profilin and profilactin complexes. Biochim Biophys Acta 1988; 967(3):391-400.

20. Janmey PA. Polyproline affinity method for purification of platelet profilin and modification with pyrene-maleimide. Methods Enzymol 1991; 196:92-9.

21. Rozycki M, Schutt, CE, Lindberg U. Affinity chromatography-based purification of profilin:actin. Methods Enzymol 1991; 196:100-18.

22. Yarmola EG, Bubb MR. Profilin: emerging concepts and lingering misconceptions. Trends Biochem Sci 2006; 31(4):197-205.

23. Barber Hernández D. Could profilin be a 'canary in a coal mine' of the increasing allergy epidemic? Int Arch Allergy Immunol 2015; 168(1):1-2.

24. de Martino M, Novembre E, Cozza G, de Marco A, Bonazza P, Vierucci A. Sensitivity to tomato and peanut allergens in children monosensitized to grass pollen. Allergy 1988; 43(3):206-13.

25. Petersen A, Vieths S, Aulepp H, Schlaak M, Becker WM. Ubiquitous structures responsible for IgE cross-reactivity between tomato fruit and grass pollen allergens. J Allergy Clin Immunol 1996; 98(4):805-15.

26. Kondo Y, Atsuo U, Tokuda R. Identification and characterization of the allergens in the tomato fruit by immunoblotting. Int Arch Allergy Immunol 2001; 126(4):294-9.

27. Reche M, Pascual CY, Vicente J, Caballero T, Martin-Munoz F, Sanchez $\mathrm{S}$, et al. Tomato allergy in children and young adults: cross-reactivity with latex and potato. Allergy 2001; 56(12):1197-201.

28. Foetisch K, Son DY, Altman F, Aulepp H, Conti A, Haustein D, et al. Tomato (Lycopersicon esculentum) allergens in pollen allergic patients. Eur Food Res Technol 2001; 213(4):259-66.

29. Willeroider M, Fuchs H, Ballmer-Weber BK, Focke M, Susani M, Thalhamer J, et al. Cloning and molecular and immunological characterization of two new food allergens, Cap a 2 and Lyc e 1, profilins from bell pepper (Capsicum annuum) and tomato (Lycopersicon esculentum). Int Arch Allergy Immunol 2003; 131(4):245-55.

30. Westphal S, Kempf W, Foetisch K, Retzek M, Vieths S, Scheurer S. Tomato profilin Lyc e 1: IgE cross-reactivity and allergenic potency. Allergy 2004; 59(5):526-32.

31. Bradford MM. A rapid and sensitive method for the quantitation of microgram quantities of protein utilizing the principle of protein-dye binding. Anal Biochem 1976; 72:248-54.

32. March SC, Parikh I, Cuatrecasas P. A simplified method for cyanogen bromide activation of agarose for affinity chromatography. Anal Biochem 1974; 60(1):149-52.

33. Sanico AM, Bochner BS, Saini SS. Skin testing methods. In: Adelman DC, Casale TB, Corren J, editors. Manual of allergy and immunology, 4th ed. Philadelphia: Lippincott Williams and Wilkins; 2002. pp. 485-6.

34. Asero R, Monsalve R, Barber D. Profilin sensitization detected in the office by skin prick test: a study of prevalence and clinical relevance of profilin as a plant food allergen. Clin Exp Allergy 2008; 38(6):1033-7.

35. Asero R, Jimeno L, Barber D. Component-resolved diagnosis of plant food allergy by SPT. Eur Ann Allergy Clin Immunol 2008; 40(4):115-21.

36. González-Mancebo E, González-de-Olano D, Trujillo MJ, Santos S, Gandolfo-Cano M, Meléndez A, et al. Prevalence of sensitiza- 
tion to lipid transfer proteins and profilins in a population of 430 patients in the south of Madrid. J Investig Allergol Clin Immunol 2011; 21(4):278-82.

37. Cho JH, Suh JD, Kim JK, Hong SC, Park IH, Lee HM. Correlation between skin-prick testing, individual specific $\operatorname{IgE}$ tests, and a multiallergen $\operatorname{IgE}$ assay for allergy detection in patients with chronic rhinitis. Am J Rhinol Allergy 2014; 28(5):388-91.

38. Goikoetxea MJ, Berroa F, Cabrera-Freitag P, Ferrer M, Núñez-Córdoba JM, Sanz ML, et al. Do skin prick test and in vitro techniques diagnose sensitization to peach lipid transfer protein and profilin equally well in allergy to plant food and pollen? J Investig Allergol Clin Immunol 2015; 25(4):283-7.

39. Asero R. Tomato allergy: clinical features and usefulness of current routinely available diagnostic methods. J Investig Allergol Clin Immunol 2013; 23(1):37-42.

40. Alvarado MI, Jimeno L, De La Torre F, Boissy P, Rivas B, Lázaro $\mathrm{MJ}$, et al. Profilin as a severe food allergen in allergic patients overexposed to grass pollen. Allergy 2014; 69(12):1610-6. 